\title{
Nonlinear mappings preserving at least one eigenvalue
}

\author{
by \\ Constantin Costara (Constanţa) and Dušan Repovš (Ljubljana)
}

\begin{abstract}
We prove that if $F$ is a Lipschitz map from the set of all complex $n \times n$ matrices into itself with $F(0)=0$ such that given any $x$ and $y$ we know that $F(x)-F(y)$ and $x-y$ have at least one common eigenvalue, then either $F(x)=u x u^{-1}$ or $F(x)=$ $u x^{t} u^{-1}$ for all $x$, for some invertible $n \times n$ matrix $u$. We arrive at the same conclusion by supposing $F$ to be of class $\mathcal{C}^{1}$ on a domain in $\mathcal{M}_{n}$ containing the null matrix, instead of Lipschitz. We also prove that if $F$ is of class $\mathcal{C}^{1}$ on a domain containing the null matrix satisfying $F(0)=0$ and $\rho(F(x)-F(y))=\rho(x-y)$ for all $x$ and $y$, where $\rho(\cdot)$ denotes the spectral radius, then there exists $\gamma \in \mathbb{C}$ of modulus one such that either $\gamma^{-1} F$ or $\gamma^{-1} \bar{F}$ is of the above form, where $\bar{F}$ is the (complex) conjugate of $F$.
\end{abstract}

1. Introduction and statement of results. Linear preserver problems deal with the question of characterizing those linear transformations on an algebra which leave invariant a certain subset, function or relation defined on the underlying algebra. The study of such transformations began with Frobenius in 1897, who characterized the linear maps on matrix algebras preserving the determinant. In 1949, Dieudonné characterized the invertible linear maps preserving the set of singular matrices. In 1959, Marcus and Moyls [7, Theorem 6] proved that if $T: \mathcal{M}_{n} \rightarrow \mathcal{M}_{n}$ is linear (with respect to complex scalars) and an eigenvalue preserver, then there exists an invertible $u \in \mathcal{M}_{n}$ such that either

$$
T(x)=u x u^{-1} \quad\left(x \in \mathcal{M}_{n}\right) \quad \text { or } \quad T(x)=u x^{t} u^{-1} \quad\left(x \in \mathcal{M}_{n}\right) .
$$

(For a fixed integer $n \geq 1$, we denote by $\mathcal{M}_{n}$ the space of all complex $n \times n$ matrices and $x^{t}$ stands for the transpose of $x \in \mathcal{M}_{n}$.) By a density argument, one can easily see that we arrive at the same conclusion by supposing $\sigma(T(x))=\sigma(x)$ for all $x \in \mathcal{M}_{n}$, where $\sigma(x)$ stands for the spectrum of $x$, that is, the set of all its eigenvalues without counting multiplicities.

One of the best known results in the theory of linear preservers is the Gleason-Kahane-Żelazko theorem (cf. e.g. [11]), which asserts that a unital linear functional $f$ defined on a (complex, unital) Banach algebra $\mathcal{A}$ is

2010 Mathematics Subject Classification: Primary 47B49; Secondary 15A18.

Key words and phrases: spectrum, Lipschitz mapping, holomorphic mapping, preserver. 
multiplicative if it preserves invertibility. For unital linear functionals, the assumption of preserving invertibility is easily seen to be equivalent to the condition that $f(x)$ belongs to the spectrum of $x$ for every $x \in \mathcal{A}$. Without assuming linearity, one needs to impose stronger preservation properties on $f$ in order to arrive at the same conclusion. Kowalski and Słodkowski proved in [6] that every functional $f$ on a Banach algebra $\mathcal{A}$ (no linearity assumed on $f$ ) with $f(0)=0$ such that the difference of the value of any two elements is contained in the spectrum of the difference of those two elements, is linear and multiplicative. Thus, we may replace the linearity assumption and the spectrum-preserving property of $f$ by a single weaker assumption and still arrive at the same conclusion.

In view of this result, it is quite natural to relax the hypothesis of linearity and try to find a criterion of Kowalski-Słodkowski type for maps defined on matrix spaces to be linear morphisms or antimorphisms. For example, Mrčun proved that the result of Marcus and Moyls [7, Theorem 6] also holds if one supposes that $T$ is only $\mathbb{R}$-linear (that is, additive and homogeneous with respect to scalars from the real field).

Lemma 1.1 ([9, Lemma 3]). Let $T: \mathcal{M}_{n} \rightarrow \mathcal{M}_{n}$ be an $\mathbb{R}$-linear mapping such that $T(x)$ and $x$ have the same spectrum for all $x \in \mathcal{M}_{n}$. Then $T$ is $\mathbb{C}$-linear (and therefore of the form (1.1)).

Mrčun then used the assertion of Lemma 1.1 and ideas from [6] to solve the following nonlinear preserver problem on matrix spaces.

Theorem 1.2 ([9, Theorem 1]). Let $F: \mathcal{M}_{n} \rightarrow \mathcal{M}_{n}$ be a Lipschitz mapping with $F(0)=0$ such that

$$
\sigma(F(x)-F(y)) \subseteq \sigma(x-y) \quad\left(x, y \in \mathcal{M}_{n}\right) .
$$

Then $T$ is of the form (1.1).

The most difficult part of the proof of [9, Theorem 1] was to show that under the assumptions of Theorem 1.2 the map $F$ is not far from being holomorphic on $\mathcal{M}_{n}$ (see the proof of [9, Lemma 4]), and this heavily depends on Lemma 1.1. The following is a generalization of [9, Lemma 3].

Lemma 1.3. Let $T: \mathcal{M}_{n} \rightarrow \mathcal{M}_{n}$ be an $\mathbb{R}$-linear mapping such that $T(x)$ and $x$ always have at least one common eigenvalue. Then $T$ is $\mathbb{C}$-linear.

With the same basic idea as the one from the proof of [9, Theorem 1], but with the use of Lemma 1.3 instead of Lemma 1.1, we obtain the following generalization of Theorem 1.2 .

TheOREM 1.4. Let $F: \mathcal{M}_{n} \rightarrow \mathcal{M}_{n}$ be a Lipschitz mapping with $F(0)=0$ such that

$$
\sigma(F(x)-F(y)) \cap \sigma(x-y) \neq \emptyset \quad\left(x, y \in \mathcal{M}_{n}\right) .
$$

Then $F$ is of the form (1.1). 
The idea to impose certain spectral properties on $F$ in order to make it holomorphic also works in the case of $\mathcal{C}^{1}$-functions. Motivated by considerations related to the study of biholomorphic maps of the open spectral unit ball of $\mathcal{M}_{n}$, Baribeau and Ransford proved that if $U$ and $V$ are open subsets of $\mathcal{M}_{n}$ and $F: U \rightarrow V$ is a bijective function of class $\mathcal{C}^{1}$ such that $\sigma(F(x))=\sigma(x)$ for every $x \in U$, then $F(x)$ is conjugate to $x$ for all $x \in U[3$, Corollary 1.2]. For example, this statement holds when $F$ is a bijective holomorphic spectrum-preserving map. When $F$ is supposed to be holomorphic, we may ask $F$ to preserve only one eigenvalue, in order to obtain the same result: it was proved in [5, Corollary 1.2] that if $U \subseteq \mathcal{M}_{n}$ is a domain containing the null matrix, $V \subseteq \mathcal{M}_{n}$ is open and $F: U \rightarrow V$ is a bijective holomorphic map such that $F(x)$ and $x$ always have at least one common eigenvalue, then $F(x)$ and $x$ are conjugate for all $x \in U$. We use this to prove a statement analogous to the one of Theorem 1.4 in the context of $\mathcal{C}^{1}$-functions.

TheOREM 1.5. Let $U \subseteq \mathcal{M}_{n}$ be a domain containing the null matrix, and $F: U \rightarrow \mathcal{M}_{n}$ a function of class $\mathcal{C}^{1}$ with $F(0)=0$. If

$$
\sigma(F(x)-F(y)) \cap \sigma(x-y) \neq \emptyset \quad(x, y \in U),
$$

then $F$ is of the form 1.1.

For $x \in \mathcal{M}_{n}$, denote by $\rho(x)$ its spectral radius. It was proved in [5, Corollary 1.4] that if $U \subseteq \mathcal{M}_{n}$ is a domain containing the null matrix, $V \subseteq \mathcal{M}_{n}$ is open and $F: U \rightarrow V$ is a bijective holomorphic map such that $F(x)$ and $x$ always have the same spectral radius, then there exists a complex number $\gamma$ of modulus one such that $\gamma^{-1} F(x)$ and $x$ are conjugate for all $x \in U$. We use this to prove the following version of Theorem 1.5 .

THeOREM 1.6. Let $U \subseteq \mathcal{M}_{n}$ be a domain containing the null matrix and $F: U \rightarrow \mathcal{M}_{n}$ be a function of class $\mathcal{C}^{1}$ with $F(0)=0$. If

$$
\rho(F(x)-F(y))=\rho(x-y) \quad(x, y \in U),
$$

then there exists $\gamma \in \mathbb{C}$ of modulus one such that either $\gamma^{-1} F$ or $\gamma^{-1} \bar{F}$ is of the form 1.1). (The map $\bar{F}: U \rightarrow \mathcal{M}_{n}$ is defined by $\bar{F}(x)=\overline{F(x)}$, the matrix obtained from $F(x)$ by entrywise complex conjugation.)

2. Proof of Lemma 1.3. We shall need the following lemma.

Lemma 2.1. Let $u \in \mathcal{M}_{n}$ be an invertible matrix such that the mapping $K: \mathcal{M}_{n} \rightarrow \mathcal{M}_{n}$ given by

$$
K(x)=\frac{x+u x u^{-1}}{2} \quad\left(x \in \mathcal{M}_{n}\right)
$$

satisfies $K\left(x^{2}\right)=K(x)^{2}$ for all $x$. Then $K$ is the identity on $\mathcal{M}_{n}$. 
Proof. For all $x$ we have

$\frac{x^{2}+u x^{2} u^{-1}}{2}=K\left(x^{2}\right)=K(x)^{2}=\frac{x^{2}}{4}+\frac{x u x u^{-1}}{4}+\frac{u x u^{-1} x}{4}+\frac{u x^{2} u^{-1}}{4}$,

and therefore

$$
\left(x-u x u^{-1}\right)^{2}=0 \quad\left(x \in \mathcal{M}_{n}\right) .
$$

In particular, $\rho\left(x-u x u^{-1}\right)=0$ for every $x$ in $\mathcal{M}_{n}$. For $x \mapsto x u$ this gives

$$
\rho(x u-u x)=0 \quad\left(x \in \mathcal{M}_{n}\right) .
$$

Using [2, Theorem 5.2.1] we conclude that $u$ belongs to the center $\mathcal{Z}\left(\mathcal{M}_{n}\right)$ of $\mathcal{M}_{n}$. Then (2.1) implies that $K(x)=x$ for all $x$.

Proof of Lemma 1.3. If $n=1$ then $T$ is the identity on $\mathbb{C}$. So suppose now that $n \geq 2$. Given $r \in \mathbb{R}$, we have $\sigma\left(T\left(e^{-i r} x\right)\right) \cap \sigma\left(e^{-i r} x\right) \neq \emptyset$, that is, $\sigma\left(e^{i r} T\left(e^{-i r} x\right)\right) \cap \sigma(x) \neq \emptyset$. Using the $\mathbb{R}$-linearity of $T$, we obtain

$$
\begin{aligned}
e^{i r} T\left(e^{-i r} x\right) & =(\cos r+i \sin r)(T(x) \cos r-T(i x) \sin r) \\
& =\frac{T(x)+T(i x) / i}{2}+e^{2 i r} \frac{T(x)-T(i x) / i}{2} .
\end{aligned}
$$

Thus

$$
\sigma(R(x)+\zeta S(x)) \cap \sigma(x) \neq \emptyset \quad\left(x \in \mathcal{M}_{n},|\zeta|=1\right),
$$

where we denoted

$$
R(x)=\frac{T(x)+T(i x) / i}{2} \quad \text { and } \quad S(x)=\frac{T(x)-T(i x) / i}{2}
$$

for $x \in \mathcal{M}_{n}$. Since $T$ is $\mathbb{R}$-linear, the same holds for $R$. One can easily check that $R(i x)=i R(x)$ for all $x$, and therefore $R$ is $\mathbb{C}$-linear.

For $x \in \mathcal{M}_{n}$, denoting by $P(\lambda)$ its characteristic polynomial we use 2.2$)$ to show that $\operatorname{det} P(R(x)+\zeta S(x))=0$ for all $\zeta$ of modulus one. Then the same holds for all complex numbers $\zeta$, and in particular $\operatorname{det} P(R(x))=0$. This means that $R(x)$ and $x$ always have at least one common eigenvalue. Using now [1, Theorem 3] we deduce that $R$ is of the form (1.1). Then without loss of generality we may suppose that $R(x)=x$ for all $x \in \mathcal{M}_{n}$. (If $R(x)=u x u^{-1}$ for all $x \in \mathcal{M}_{n}$ then we work with $x \mapsto u^{-1} T(x) u$ instead of $T$, and if $R(x)=u x^{t} u^{-1}$ for all $x \in \mathcal{M}_{n}$ then we work with $x \mapsto\left(u^{-1} T(x) u\right)^{t}$ instead of $T$.) This means that

$$
T(x)+T(i x) / i=2 x \quad\left(x \in \mathcal{M}_{n}\right) .
$$

Define now the mapping $W: \mathcal{M}_{n} \rightarrow \mathcal{M}_{n}$ by putting

$$
W(x)=T\left(\frac{x+x^{*}}{2}\right)+i T\left(\frac{x-x^{*}}{2 i}\right) \quad\left(x \in \mathcal{M}_{n}\right) .
$$

One can easily check that $W$ is $\mathbb{C}$-linear. Moreover, for an arbitrary Hermitian matrix $x$ we have $W(x)=T(x)$, and therefore $\sigma(W(x)) \cap \sigma(x) \neq \emptyset$. 
Then [1, Corollary 3] implies that $W$ is an eigenvalue-preserver, so by [7, Theorem 6] there exists an invertible matrix $u$ such that

$$
W(x)=u x u^{-1} \quad\left(x \in \mathcal{M}_{n}\right) \quad \text { or } \quad W(x)=u x^{t} u^{-1} \quad\left(x \in \mathcal{M}_{n}\right) .
$$

Suppose that the first case occurs in 2.4 . Then $T(x)=u x u^{-1}$ for all $x$ satisfying $x=x^{*}$. Given an arbitrary $x \in \mathcal{M}_{n}$, writing it as $x=x_{1}+i x_{2}$ with $x_{1}, x_{2}$ Hermitian matrices and using (2.3) we get

$$
\begin{aligned}
T(x) & =T\left(x_{1}\right)+T\left(i x_{2}\right)=u x_{1} u^{-1}+i\left(2 x_{2}-T\left(x_{2}\right)\right) \\
& =u\left(x_{1}-i x_{2}\right) u^{-1}+2 i x_{2},
\end{aligned}
$$

and therefore

$$
T(x)=x+\left(u x^{*} u^{-1}-x^{*}\right) \quad\left(x \in \mathcal{M}_{n}\right) .
$$

Since $\sigma(T(\lambda x) / \lambda) \cap \sigma(x) \neq \emptyset$ for all $\lambda \in \mathbb{C} \backslash\{0\}$, it follows that $\sigma(x+$ $\left.\zeta\left(u x^{*} u^{-1}-x^{*}\right)\right) \cap \sigma(x) \neq \emptyset$ for all $x \in \mathcal{M}_{n}$ and $|\zeta|=1$ in $\mathbb{C}$. Once more, for $x \in \mathcal{M}_{n}$ denoting by $P(\lambda)$ its characteristic polynomial we have $\operatorname{det} P(x+$ $\left.\zeta\left(u x^{*} u^{-1}-x^{*}\right)\right)=0$ for all $\zeta \in \mathbb{C}$. Thus $\sigma\left(x+\zeta\left(u x^{*} u^{-1}-x^{*}\right)\right) \cap \sigma(x) \neq \emptyset$ for all $x \in \mathcal{M}_{n}$ and $\zeta \in \mathbb{C}$. Taking $\zeta=1 / 2$ we obtain $\sigma\left(x+\left(u x^{*} u^{-1}-x^{*}\right) / 2\right) \cap$ $\sigma(x) \neq \emptyset$ for all $x \in \mathcal{M}_{n}$. Define $K: \mathcal{M}_{n} \rightarrow \mathcal{M}_{n}$ by putting

$$
K(x)=x+\left(u x u^{-1}-x\right) / 2=\left(x+u x u^{-1}\right) / 2 .
$$

Then $K$ is $\mathbb{C}$-linear and $\sigma(K(x)) \cap \sigma(x) \neq \emptyset$ for all $x \in \mathcal{M}_{n}$ satisfying $x=x^{*}$. Using once more [1, Corollary 3], we find that $K$ is a Jordan morphism. Hence Lemma 2.1 implies that $K(x)=x$ for all $x$. Then $u x u^{-1}=x$ for all $x$, and (2.5) gives $T(x)=x$ for all $x$. In particular, $T$ is $\mathbb{C}$-linear.

Let us now prove that the second case in $(2.4)$ cannot occur. If $W(x)=$ $u x^{t} u^{-1}$ for all $x$, then exactly as in the proof of (2.5) we deduce that $T(x)=$ $x+\left(u \bar{x} u^{-1}-x^{*}\right)$ for all $x$, where $\bar{x}$ is the matrix obtained from $x$ by entrywise conjugation. Once more, $\sigma(T(\lambda x) / \lambda) \cap \sigma(x) \neq \emptyset$ for all nonzero $\lambda$ gives $\sigma\left(x+\left(u \bar{x} u^{-1}-x^{*}\right) / 2\right) \cap \sigma(x) \neq \emptyset$ for all $x \in \mathcal{M}_{n}$. Define $J: \mathcal{M}_{n} \rightarrow \mathcal{M}_{n}$ by putting

$$
J(x)=x+\left(u x^{t} u^{-1}-x\right) / 2=\left(x+u x^{t} u^{-1}\right) / 2 .
$$

Then $J$ is $\mathbb{C}$-linear and $\sigma(J(x)) \cap \sigma(x) \neq \emptyset$ for all $x \in \mathcal{M}_{n}$ satisfying $x=x^{*}$. It follows by [1, Corollary 3] that $J$ is either a morphism or an antimorphism. In both cases we have $\operatorname{Tr}(J(x y))=\operatorname{Tr}(J(x) J(y))$ for all $x, y \in \mathcal{M}_{n}$, where $\operatorname{Tr}(\cdot)$ denotes the usual trace on $\mathcal{M}_{n}$. Using the properties of the trace now gives

$$
\operatorname{Tr}\left(x\left(2 y-u y^{t} u^{-1}-u^{t} y^{t}\left(u^{t}\right)^{-1}\right)\right)=0 \quad\left(x, y \in \mathcal{M}_{n}\right) .
$$

It follows that $y=\left(u y^{t} u^{-1}+u^{t} y^{t}\left(u^{t}\right)^{-1}\right) / 2$ for all $y \in \mathcal{M}_{n}$, which can be rewritten as

$$
u^{-1} z^{t} u=\frac{z+\left(u^{-1} u^{t}\right) z\left(u^{-1} u^{t}\right)^{-1}}{2} \quad\left(z \in \mathcal{M}_{n}\right) .
$$


Since $z \mapsto u^{-1} z^{t} u$ is a Jordan morphism on $\mathcal{M}_{n}$, using Lemma 2.1 we deduce that $u^{-1} z^{t} u=z$ for all $z$ in $\mathcal{M}_{n}$. Then $a b=\left(u^{-1} a^{t} u\right)\left(u^{-1} b^{t} u\right)=u^{-1}(b a)^{t} u=$ $b a$ for all $a, b \in \mathcal{M}_{n}$, and we arrive at a contradiction.

We now use Lemma 1.3 to give a new proof of a special case of a result of Bhatia, Šemrl and Sourour on maps on matrices that preserve the spectral radius distance [4, Theorem 1.1].

COROLlary 2.2. Let $T: \mathcal{M}_{n} \rightarrow \mathcal{M}_{n}$ be an $\mathbb{R}$-linear mapping such that $\rho(T(x))=\rho(x)$ for all $x$. Then either $T$ or $\bar{T}$ is $\mathbb{C}$-linear.

Proof. Even though $T$ is not supposed to be $\mathbb{C}$-linear, it shares all the basic properties of spectral isometries [8]. Let us first prove that $T$ is bijective. Since $T$ is $\mathbb{R}$-linear and $\mathcal{M}_{n}$ is of finite dimension over $\mathbb{R}$, it suffices to prove that $T$ is injective. Since $T$ is additive, we must prove that $T(x)=0$ implies $x=0$. This follows from the identity

$$
\rho(y)=\rho(T(y))=\rho(T(y)+T(x))=\rho(T(y+x))=\rho(y+x),
$$

which is valid for all $y \in \mathcal{M}_{n}$, and from the characterization of the radical given by [2, Theorem 5.3.1].

We now prove that $T$ sends the center $\mathcal{Z}\left(\mathcal{M}_{n}\right)$ of $\mathcal{M}_{n}$ into itself. By [2, Theorem 5.2.2], we have $x \in \mathcal{Z}\left(\mathcal{M}_{n}\right)$ if and only if there exists $M>0$ such that $\rho(x+y) \leq M(1+\rho(y))$ for all $y \in \mathcal{M}_{n}$. So if $x=\lambda I_{n}$ for some $\lambda \in \mathbb{C}$, then for all $y \in \mathcal{M}_{n}$ we have

$$
\rho(T(x)+y)=\rho(T(x)+T(z))=\rho(x+z) \leq M(1+\rho(z))=M(1+\rho(y)) .
$$

(Since $T$ is bijective, there exists $z$ such that $T(z)=y$.) Thus $T(x) \in \mathcal{Z}\left(\mathcal{M}_{n}\right)$. In particular, $T\left(I_{n}\right)=\mu I_{n}$ for some $|\mu|=1$. So by multiplying $T$ with $\mu^{-1}$, we may suppose that $T\left(I_{n}\right)=I_{n}$. Also, $T\left(i I_{n}\right)=\zeta I_{n}$ for some complex number $\zeta$ with $|\zeta|=1$. Therefore

$$
\sqrt{r^{2}+s^{2}}=\rho\left((s+r i) I_{n}\right)=\rho\left(T\left((s+r i) I_{n}\right)\right)=\rho\left((s+\zeta r) I_{n}\right)=|s+r \zeta|
$$

for all $r, s \in \mathbb{R}$ implies that either $\zeta=i$ or $\zeta=-i$. In the first case we have $T\left(i I_{n}\right)=i I_{n}$ and hence, by the $\mathbb{R}$-linearity of $T$, we get $T\left(\lambda I_{n}\right)=\lambda I_{n}$ for all $\lambda \in \mathbb{C}$. In the second case we obtain $\overline{T\left(\lambda I_{n}\right)}=\lambda I_{n}$ for all $\lambda \in \mathbb{C}$.

We complete the proof by showing, for example, that if $T\left(\lambda I_{n}\right)=\lambda I_{n}$ for all $\lambda \in \mathbb{C}$ then $T$ preserves the peripheral spectrum. If this is true, then $x$ and $T(x)$ always have at least one common eigenvalue and Lemma 1.3 implies that $T$ is $\mathbb{C}$-linear. So let $x \in \mathcal{M}_{n}$ and consider $\lambda \in \sigma(x)$ such that $\rho(x)=|\lambda|$. Then

$$
\rho\left(T(x)+\lambda I_{n}\right)=\rho\left(T\left(x+\lambda I_{n}\right)\right)=\rho\left(x+\lambda I_{n}\right)=2|\lambda|=2 \rho(x),
$$

and hence there exists $\alpha \in \sigma(T(x))$ such that $|\alpha+\lambda|=2|\lambda|$. Since $|\alpha| \leq$ $\rho(T(x))=|\lambda|$, it follows that $\alpha=\lambda$. Therefore, the peripheral spectrum of $x$ lies inside the peripheral spectrum of $T(x)$. Since $T^{-1}$ also preserves the 
spectral radius and $T^{-1}\left(\lambda I_{n}\right)=\lambda I_{n}$ for all $\lambda \in \mathbb{C}$, we may conclude that $x$ and $T(x)$ always have the same peripheral spectrum.

Corollary 2.3. Suppose that $T: \mathcal{M}_{n} \rightarrow \mathcal{M}_{n}$ is an $\mathbb{R}$-linear mapping which preserves the spectral radius. There exists then a complex number $\gamma$ of modulus one such that either $\gamma^{-1} T$ or $\gamma^{-1} \bar{T}$ is of the form 1.1.

Proof. We use Corollary 2.2 and we suppose, for example, that $T$ is $\mathbb{C}$-linear. As we have seen in the proof of Corollary 2.2, since $\rho(T(x))=\rho(x)$ for all $x$ we find that $T\left(I_{n}\right)=\gamma I_{n}$ for some $|\gamma|=1$. Thus $\gamma^{-1} T: \mathcal{M}_{n} \rightarrow \mathcal{M}_{n}$ is a unital spectral isomorphism, and therefore a Jordan isomorphism 8 , Corollary 5]. Thus $\gamma^{-1} T$ is of the form (1.1).

3. Proofs for the case of Lipschitz functions. Let us first recall that the real (respectively, complex) differential of a function $F: \mathcal{M}_{n} \rightarrow \mathcal{M}_{m}$ at a point $x_{0} \in \mathcal{M}_{n}$ is a mapping $(D F)_{x_{0}}: \mathcal{M}_{n} \rightarrow \mathcal{M}_{m}$ which is linear with respect to the real (respectively, complex) scalars, and such that

$$
\lim _{x \rightarrow 0} \frac{\left\|F\left(x_{0}+x\right)-F\left(x_{0}\right)-(D F)_{x_{0}}(x)\right\|}{\|x\|}=0 .
$$

(We work with the operator norm on $\mathcal{M}_{n}$.)

We shall use the following lemmas. The first one is a consequence of a result of Rademacher [12, p. 50].

Lemma 3.1. Let $n$ be a positive integer and $f: \mathcal{M}_{n} \rightarrow \mathbb{C}$ a Lipschitz function. Then $f$ has real differentials a.e. with respect to the Lebesgue measure $m$ on $\mathcal{M}_{n}$.

The second one is a generalization of a result from [6, Lemma 3.2].

Lemma 3.2 ([9, Lemma 4]). Let $f: \mathcal{M}_{n} \rightarrow \mathbb{C}$ be a Lipschitz function and assume that $f$ has complex differentials a.e. with respect to the Lebesgue measure on $\mathcal{M}_{n}$. Then for all $a, b \in \mathcal{M}_{n}$, the function $f_{a, b}: \mathbb{C} \rightarrow \mathbb{C}$ given by

$$
f_{a, b}(z)=f(a+b z) \quad(z \in \mathbb{C})
$$

is affine.

Proof of Theorem 1.4. We use the ideas from [9, proof of Theorem 1]. Let $F_{s, k}: \mathcal{M}_{n} \rightarrow \mathbb{C}$ be given by

$$
F_{s, k}(x)=(F(x))_{s, k} \quad\left(x \in \mathcal{M}_{n}\right)
$$

for $s, k=1, \ldots, n$. Since $F$ is Lipschitz this implies that all the mappings $F_{s, k}$ are Lipschitz. By Lemma 3.1, the function $F_{s, k}$ has real differentials on $\mathcal{M}_{n} \backslash Z_{s, k}$ with $m\left(Z_{s, k}\right)=0$. Denoting $Z=\bigcup_{s, k=1}^{n} Z_{s, k}$, we see that $m(Z)=0$ and the real differential $\left(D T_{s, k}\right)_{x}$ exists for all $s, k=1, \ldots, n$ and for all $x \in \mathcal{M}_{n} \backslash Z$. Then for all $x \in \mathcal{M}_{n} \backslash Z$ we conclude that $F: \mathcal{M}_{n} \rightarrow \mathcal{M}_{n}$ 
has real differential $(D F)_{x}: \mathcal{M}_{n} \rightarrow \mathcal{M}_{n}$ at $x$ given by

$$
(D F)_{x}(u)=\left(\left(D F_{s, k}\right)_{x}(u)\right)_{s, k=1}^{n} \quad\left(u \in \mathcal{M}_{n}\right) .
$$

Given $x \in \mathcal{M}_{n} \backslash Z$, we see by $(1.3)$ that for all strictly positive integers $m$,

$$
\sigma\left(\frac{F(x+u / m)-F(x)}{1 / m}\right) \cap \sigma(u) \neq \emptyset \quad\left(u \in \mathcal{M}_{n}\right) .
$$

For $m \rightarrow \infty$, using the continuity properties for the spectrum it follows that

$$
\sigma\left((D F)_{x}(u)\right) \cap \sigma(u) \neq \emptyset \quad\left(x \in \mathcal{M}_{n} \backslash Z ; u \in \mathcal{M}_{n}\right) .
$$

Then Lemma 1.3 shows that $(D F)_{x}: \mathcal{M}_{n} \rightarrow \mathcal{M}_{n}$ is $\mathbb{C}$-linear, and hence $\left(D F_{s, k}\right)_{x}: \mathcal{M}_{n} \rightarrow \mathbb{C}$ are $\mathbb{C}$-linear mappings for all $x \in \mathcal{M}_{n} \backslash Z$ and $s, k=$ $1, \ldots, n$. By Lemma 3.2 , fixing any $a, b \in \mathcal{M}_{n}$ we find that $\lambda \mapsto F_{s, k}(a+\lambda b)$ is affine, and hence the same must hold for $F_{a, b}: \mathbb{C} \rightarrow \mathcal{M}_{n}$ given by

$$
F_{a, b}(\lambda)=F(a+\lambda b) \quad(\lambda \in \mathbb{C}) .
$$

Thus $F_{a, b}(\lambda)=\lambda\left(F_{a, b}(1)-F_{a, b}(0)\right)+F_{a, b}(0)$ for all $\lambda \in \mathbb{C}$, that is,

$$
F(a+\lambda b)=\lambda(F(a+b)-F(a))+F(a) \quad\left(\lambda \in \mathbb{C}, a, b \in \mathcal{M}_{n}\right) .
$$

Taking $a=0$ in (3.1) we obtain $F(\lambda b)=\lambda F(b)$ for every $\lambda \in \mathbb{C}$ and for every $b \in \mathcal{M}_{n}$. Taking $\lambda=1$ and replacing $b$ by $(c-a) / 2$ in (3.1) we get $F(c)=F(a+c)-F(a)$, which yields

$$
F(a+c)=F(a)+F(c) \quad\left(a, c \in \mathcal{M}_{n}\right) .
$$

Thus $F: \mathcal{M}_{n} \rightarrow \mathcal{M}_{n}$ is $\mathbb{C}$-linear.

For $y=0$ in 1.3 we see that $\sigma(F(x)) \cap \sigma(x) \neq \emptyset$ for all $x \in \mathcal{M}_{n}$. Since $F$ is $\mathbb{C}$-linear, it follows from [1, Theorem 2] that $F$ is indeed of the form (1.1).

4. Proofs for the case of $\mathcal{C}^{1}$-functions. For conjugate matrices $x$ and $y$ in $\mathcal{M}_{n}$, we shall write $x \sim y$.

Proof of Theorem 1.5. By hypothesis, the function $F$ has real differential $(D F)_{x}$ at every point $x$ of $U$. The proof of Theorem 1.4 shows that in fact $(D F)_{x}: \mathcal{M}_{n} \rightarrow \mathcal{M}_{n}$ is $\mathbb{C}$-linear for all $x$ in $U$. This implies that $F$ is holomorphic on $U$ [10, Theorem 1.2]. By (1.4) we also have

$$
\sigma\left((D F)_{x}(v)\right) \cap \sigma(v) \neq \emptyset \quad\left(x \in U, v \in \mathcal{M}_{n}\right),
$$

and using [1, Theorem 2] we deduce that $(D F)_{x}$ is of the form (1.1) for all $x$ in $U$. In particular $(D F)_{0}: \mathcal{M}_{n} \rightarrow \mathcal{M}_{n}$ is invertible, and by the inverse mapping theorem there exists $\delta>0$ such that denoting by $W$ the open ball (with respect to the operator norm) of center 0 and radius $\delta$ in $\mathcal{M}_{n}$ we find that $W \subseteq U, F(W) \subseteq \mathcal{M}_{n}$ is open and $F: W \rightarrow F(W)$ is biholomorphic. For $x$ with $\|x\|<\delta / 2$, let us define $\varphi_{x}:\{h:\|h\|<\delta / 2\} \rightarrow \mathcal{M}_{n}$ by putting $\varphi_{x}(h)=F(x+h)-F(x)$. By (1.4), we have $\sigma\left(\varphi_{x}(h)\right) \cap \sigma(h) \neq \emptyset$ for all 
$\|h\|<\delta / 2$. Using [5, Corollary 1.2] we obtain $\varphi_{x}(h) \sim h$ for $\|h\|<\delta / 2$. That is, $F(x+h)-F(x) \sim h$ for all $x, h$ with $\|x\|,\|h\|<\delta / 2$.

There exists an invertible $u \in \mathcal{M}_{n}$ such that either $(D F)_{0}(v)=u v u^{-1}$ for all $v$, or $(D F)_{0}(v)=u v^{t} u^{-1}$ for all $v$. If the first case occurs, we consider $G: U \rightarrow \mathcal{M}_{n}$ given by $G(x)=u^{-1} F(x) u$, and in the second case we consider $G: U \rightarrow \mathcal{M}_{n}$ given by $G(x)=\left(u^{-1} F(x) u\right)^{t}$. In both cases, we obtain a holomorphic function $G$ on $U$ such that $G(0)=0, G^{\prime}(0)$ is the identity on $\mathcal{M}_{n}$, and

$$
G(x+h)-G(x) \sim h \quad(\|x\|,\|h\|<\delta / 2) .
$$

We finish the proof by showing that $G$ with the above properties must be the identity on $U$. To see this, first observe that by taking $x=0$ in (4.1) we have $G(h) \sim h$ for all $h$ with $\|h\|<\delta / 2$. Then $G(h)^{2} \sim h^{2}$ for all such $h$, and thus $\operatorname{Tr}(G(h))=\operatorname{Tr}(h)$ and $\operatorname{Tr}\left(G(h)^{2}\right)=\operatorname{Tr}\left(h^{2}\right)$ for $\|h\|<\delta / 2$. Also, for $\|x\|,\|y\|<\delta / 4$ by (4.1) we have $(G(x)-G(y)) \sim(x-y)$. This gives $(G(x)-G(y))^{2} \sim(x-y)^{2}$, and in particular

$$
\operatorname{Tr}\left((G(x)-G(y))^{2}\right)=\operatorname{Tr}\left((x-y)^{2}\right) .
$$

For $\|x\|,\|y\|<\delta / 4$, since $\operatorname{Tr}\left(G(x)^{2}\right)=\operatorname{Tr}\left(x^{2}\right)$ and $\operatorname{Tr}\left(G(y)^{2}\right)=\operatorname{Tr}\left(y^{2}\right)$ it follows that $\operatorname{Tr}(G(x) G(y))=\operatorname{Tr}(x y)$. Thus for all $x$ with $\|x\|<\delta / 4$ and for all $y$ we have

$$
\operatorname{Tr}(G(x) G(\lambda y))=\lambda \operatorname{Tr}(x y)
$$

for all $\lambda$ in a neighborhood of $0 \in \mathbb{C}$. Since $G^{\prime}(0)$ is the identity, it follows that $G(\lambda y)=\lambda y+\sum_{j=2}^{\infty} \lambda^{j} b_{j}$ for $\lambda$ near 0 , for some $\left(b_{j}\right)_{j \geq 2} \subseteq \mathcal{M}_{n}$. By equating the coefficients of $\lambda$ in 4.2 we get

$$
\operatorname{Tr}((G(x)-x) y)=0 \quad\left(\|x\|<\delta / 4, y \in \mathcal{M}_{n}\right) .
$$

Thus $G(x)=x$ for $\|x\|<\delta / 4$, and by the identity principle for holomorphic functions we conclude that $G(x)=x$ for all $x \in U$.

Proof of Theorem 1.6. Using the continuity of the spectral radius, from (1.5) we obtain

$$
\rho\left((D F)_{x}(v)\right)=\rho(v) \quad\left(x \in U, v \in \mathcal{M}_{n}\right) .
$$

By Corollary 2.2, given any $x \in U$ we find that either $(D F)_{x}$ is $\mathbb{C}$-linear, or $(D F)_{x}$ is $\overline{\mathbb{C}}$-linear. Defining

$$
U_{1}=\left\{x \in U:(D F)_{x} \text { is } \mathbb{C} \text {-linear }\right\}, \quad U_{2}=\left\{x \in U:(D F)_{x} \text { is } \overline{\mathbb{C}} \text {-linear }\right\},
$$

we conclude that $U_{1}, U_{2} \subseteq U$ are disjoint subsets (by Corollary 2.3 , we see that $(D F)_{x}: \mathcal{M}_{n} \rightarrow \mathcal{M}_{n}$ is always invertible, and hence we cannot have $(D F)_{x}=0$ for some $x$ in $\left.U\right)$ with $U_{1} \cup U_{2}=U$. Also, since $F$ is of class $\mathcal{C}^{1}$ it follows that $U_{1}, U_{2} \subseteq U$ are (possibly empty) closed subsets. Since $U$ is a domain, one of the $U_{j}$ is empty. So suppose, for example, that $U=U_{1}$. (In 
the remaining case, we work with $\bar{F}$ instead of $F$.) Then [10, Theorem 1.2] shows that $F$ is holomorphic on $U$.

We now proceed as in the proof of Theorem 1.5 , we shall use [5, Corollary 1.4] instead of [5, Corollary 1.2]. For $0 \in U$, by Corollary 2.3 there exists a complex number $\gamma$ of modulus one and an invertible $u$ such that either $\gamma^{-1}(D F)_{0}(v)=u v u^{-1}$ for all $v$, or $\gamma^{-1}(D F)_{0}(v)=u v^{t} u^{-1}$ for all $v$. Suppose, for example, that the first case occurs, and define $G: U \rightarrow \mathcal{M}_{n}$ by putting $G(x)=\gamma^{-1} u^{-1} F(x) u$. Then $G$ is holomorphic, $G(0)=0, G^{\prime}(0)=$ the identity of $\mathcal{M}_{n}$, and 1.5 gives

$$
\rho(G(x)-G(y))=\rho(x-y) \quad(x, y \in U) .
$$

Since $G^{\prime}(0)$ is invertible, there exists $\delta>0$ such that $G(W) \subseteq \mathcal{M}_{n}$ is open and $G: W \rightarrow G(W)$ is biholomorphic, where $W$ is the open ball with center 0 and radius $\delta$ in $\mathcal{M}_{n}$. Taking $y=0$ in 4.3 we have $\rho(G(x))=\rho(x)$ for all $x \in U$. Using [5, Corollary 1.4], we establish the existence of $\xi \in \mathbb{C}$ of modulus one such that $G(x) \sim \xi x$ for $\|x\|<\delta$. Then $G\left(\lambda I_{n}\right)=\xi \lambda I_{n}$ for $|\lambda|<\delta$, and taking the derivative with respect to $\lambda$ and using that $G^{\prime}(0)$ is the identity, we get $\xi=1$. Thus

$$
G(x) \sim x \quad(\|x\|<\delta) .
$$

For $\|x\|<\delta / 2$ in $\mathcal{M}_{n}$, define $\varphi_{x}:\{h:\|h\|<\delta / 2\} \rightarrow \mathcal{M}_{n}$ by putting $\varphi_{x}(h)=G(x+h)-G(x)$. By 4.3), we have $\rho\left(\varphi_{x}(h)\right)=\rho(h)$ for all $\|h\|<\delta / 2$. Using [5, Corollary 1.4] we verify the existence of $\xi_{x} \in \mathbb{C}$ of modulus one such that $\varphi_{x}(h) \sim \xi_{x} h$ for $\|h\|<\delta / 2$. That is, $G(x+h)-G(x) \sim \xi_{x} h$ for all $h$ with $\|h\|<\delta / 2$. For $h=(\delta / 4) I_{n}$ we get $G\left(x+(\delta / 4) I_{n}\right)-G(x)=$ $\xi_{x}(\delta / 4) I_{n}$. Taking the trace and using (4.4) we deduce that $\xi_{x}=1$. Therefore, $G(x+h)-G(x) \sim h$ for all $x, h$ with $\|x\|,\|h\|<\delta / 2$, and from this, exactly as in the final part of the proof of Theorem 1.5 . we conclude that $G$ is the identity on $U$.

Acknowledgements. Research of the first author was supported by CNCSIS-UEFISCSU, project no. 24/11.08.2010, PN II-RU Code 300/2010. The second author was supported by the Slovenian Research Agency grants P1-0292-0101, J1-9643-0101 and J1-2057-0101. We acknowledge the referee for comments and suggestions.

\section{References}

[1] S. Akbari and M. Aryapoor, On linear transformations preserving at least one eigenvalue, Proc. Amer. Math. Soc. 132 (2004), 1621-1625.

[2] B. Aupetit, A Primer on Spectral Theory, Springer, New York, 1991.

[3] L. Baribeau and T. Ransford, Non-linear spectrum-preserving maps, Bull. London Math. Soc. 32 (2000), 8-14. 
[4] R. Bhatia, P. Šemrl and A. R. Sourour, Maps on matrices that preserve the spectral radius distance, Studia Math. 134 (1999), 99-110.

[5] C. Costara and T. Ransford, On local irreducibility of the spectrum, Proc. Amer. Math. Soc. 135 (2007), 2779-2784.

[6] S. Kowalski and Z. Słodkowski, A characterization of multiplicative linear functionals in Banach algebras, Studia Math. 67 (1980), 215-223.

[7] M. Marcus and B. N. Moyls, Linear transformations on algebras of matrices, Canad. J. Math. 11 (1959), 61-66.

[8] M. Mathieu and A. R. Sourour, Hereditary properties of spectral isometries, Arch. Math. 82 (Basel) (2004), 222-229.

[9] J. Mrčun, Lipschitz spectrum preserving mappings on algebras of matrices, Linear Algebra Appl. 215 (1999), 113-120.

[10] R. M. Range, Holomorphic Functions and Integral Representations in Several Complex Variables, Springer, New York, 1986.

[11] W. Żelazko, Banach Algebras, PWN, Warszawa, and Elsevier, Amsterdam, 1973.

[12] W. P. Ziemer, Weakly Differentiable Functions, Springer, New York, 1989.

Constantin Costara (corresponding author)

Faculty of Mathematics and Informatics

Ovidius University

Mamaia Blvd. 124

Constanţa, Romania

E-mail: cdcostara@univ-ovidius.ro
Dušan Repovš

Faculty of Mathematics and Physics and Faculty of Education University of Ljubljana P.O. Box 2964

Ljubljana 1001, Slovenia E-mail: dusan.repovs@guest.arnes.si

Received January 14, 2010

Revised version May 31, 2010 